\title{
Mechanical dilatancy and contraction effect of contaminated soil solidified by organic solvent
}

\author{
CHENG Feng ${ }^{1,2}$, WEI Cheng ${ }^{2 *}$, CHEN Aijun ${ }^{1}$, WU Di ${ }^{1}$, CHEN Junhua $^{1}$, SU Chunhui ${ }^{1}$ \\ ${ }^{1}$ School of Architecture and Transportation Engineering, Guilin University of Electronic Technology, Guilin, 541004, China \\ ${ }^{2}$ China Nonferrous Metal (Guilin) Geology And Mining Co. Ltd, Guilin, 541004, China
}

\begin{abstract}
The mix proportion experiment was applied to investigate the mix proportion scheme of organic soil mass curing agent, and the functional mechanism of organic solvent fused with soil mass was analyzed. Under conditions of low confining pressure $(90 \sim 240 \mathrm{kPa})$ and relative higher confining pressure $(600 \sim 1200 \mathrm{kPa})$, the consolidated drained triaxial shear test was applied to compare the mechanical dilatancy and contraction effect of undisturbed soil, contaminated soil and consolidated soil, and draw the mechanical volumetric strain characteristic curves. Then the volumetric strain characteristics of dilatancy and contraction behavior and their peak strength change rules of heavy metal contaminated soil, before and after consolidation, were analyzed. According to Pietruszczak's hardening rule, the two yield surfaces volumetric strain model of heavy metal contaminated soil was established. Results indicate that heavy metal contaminated soil shows dilatancy property under the low confining condition and contraction property under high confining condition, while peak strength decreases obviously. Soil consolidation effectively improves the dilatancy volumetric strain characteristic under low confining condition, contraction volumetric characteristic under medium to high confining condition, and the peak strength increased significantly. This model makes up for the defect that the traditional single yield surface model cannot describe the critical strain state of dilatancy and contraction, and it reasonably reflects the volumetric strain change process of heavy metal contaminated soil.
\end{abstract}

\section{Introduction}

Heavy metal contaminated soil comprehensively distributes in many provinces with rich non-ferrous resources in China, such as Guangxi, Guizhou, Yunnan and so on. it has been usually treated asundisturbed soil during engineering construction, which leads to many engineering accidents ${ }^{[1]}$. Currently, the studies of heavy metal contaminated soil are mainly focused on the influencing mechanisms, while, so far, the investigations of curing mechanical properties of contaminated soil and its improved effects are few. Pan et al. ${ }^{[1,3-6]}$ reported that heavy metals are easily adhered to rock soil and cause hydrolytic mineralization reaction after invading. Especially these heavy metals destroy the internal structure of soil during the process of corrosion and migration, which enormously decreases the cementation force of soil particles. Experimental results of $\mathrm{Cao}^{[2,5-7]}$ indicate that after being adsorbed and migrated of heavy metals, the soil mass of contaminated soil will harden and the soil particles will enlarge. The strain relation is appeared as displacement and slipping between particles, and the constitutive relation is familiar with the coarsegrained soil. The conventional constitutive relation model is consequently not suitable to describe the deformation of contaminated soil. Hence, Cha et al. ${ }^{[3,8-10]}$ issued that when the conventional single yield surface model is applied to describe the strain relation of displacing and slipping, it has certain defects and needs to be improved. On the aspect of materials consolidation, current investigation achievements are mainly the inorganic curing materials. Guo et al. ${ }^{[5,6-9]}$ found that under the effect of curing agents such as concrete and lime, the mechanical strength and stability of heavy metal contaminated soil are improved effectively, but the structure of soil mass is destroyed and the elasticity is weakened. The cost of curing is higher and can not be extended to apply. Huang et al. ${ }^{[5]}$ found that the organic curing agents have better moisture keeping ability and can effectively restrain the migration of heavy metals in soil according to the metal migration experiments. Meanwhile, the organic curing agents have inhibition effect on the corrosion process of contaminants, which significantly improve the physical properties of soil mass, but the mix proportion scheme and the curing effect of organic curing agents still need to be investigated further.

In order to further investigate the mechanical characteristics change the rule of contaminated soil and the reliable curing agents of soil mass, this article studies the mechanical characteristics and change rule of undisturbed soil, contaminated soil, and solidified soil using drained triaxial shear test comparison experiment. Then, the organic materials that are suitable to consolidate the contaminated soil, the mix proportion, and the consolidation effect are explored. Next, the strength characteristics and volumetric strain change rule of heavy

\footnotetext{
* Corresponding author: Email: balckfire513@126.com.
} 
metal contaminated soil before and after curing with organic curing agents are investigated. Based on the double yield surface model of the coarse particle soil, applying the corrected Cambridge model as compression yield surface function and combining the Stolle yield function $^{[4]}$ and Pietruszczak rule as shear yield surface function, this paper establishes the softening elastoplastic two-yield surface constitutive strain model suitable to heavy metal contaminated soil. The achievement of this study provides a reliable theoretical basis and materials selection method for governing engineers of contaminated soil.

\section{Organic solidified soil experiment}

\subsection{Selection of basic research objects}

The organic curing agent is a highly condensed organic solvent that has a strong capacity of displacement and solubility ${ }^{[5,6]}$. Organic agents can effectively prevent the damage of heavy metal elements to the double electric layer structure between soil particles and the damage of the hydration membrane. Also, it can restrict the ion exchange activity of colloidal minerals and immersed elements of soil, and prevent the corrosion of cementing materials in the soil. The chemical force produced by curing agent can block the diffusing pathway of heavy metal cation and repair thickness of the double electric layer of soil particles, which keeps the ionization equilibrium of colloidal minerals and improve the cementation force ${ }^{[6]}$. The working principle of the curing agents can be seen in Figure 1.

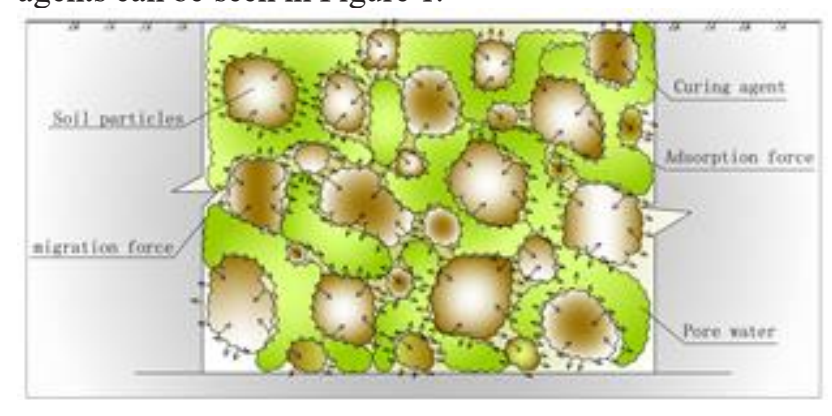

Fig. 1. Working scheme diagram of curing agent.

The soil solidified by curing agents is different from normal agents, such as lime and concrete. The formed tabular structure after being consolidated will not destroy the natural characteristics of soil mass ${ }^{[6,7]}$ and has better capacity on elastoplastic. Especially after consolidation, the heavy metal contaminated soil reduces the hardening effect, is not easy to crack, and is better in the waterretaining property. It also effectively improves shearing force, compactibility, and stability.

\subsection{Experimental material}

\subsubsection{Organic curing materials}

The organic curing material used in the experiment is mainly the epoxy curing agent (4.4' diamino diphenyl methane), epoxy curing accelerator (Tetraethylamine bromide), and diluter (Diethylene glycol-ether). These several materials have their specific advantages, among which epoxy curing agent can effectively restrain hydration membrane from being destroyed and reduce corrosion of colloidal minerals, epoxy curing accelerator can effectively promote cementing of soil particles and enhance cohesive force, and dilutor controls migration of heavy metals and reduces their capacity of corrosion. The materials are used after mix proportion and will form the complete curing matrix that improves micro structure function and shows better application effects on the aspect of mechanical properties. The materials used in organic curing agents are environmentally friendly materials ${ }^{[7]}$, which are of excellent stability, difficult to drain, noncorrosive, and will not make secondary contamination to the soil mass. In addition, after being acted with organic curing agents, soil mass obtains better adhesive property and water-retaining property, and can effectively prevent soil erosion.

\subsubsection{Soil samples of experiment}

The samples of undisturbed soil and contaminated soil were sampled from the same soft soil area of Lead-zinc mine near North Mountain, Hechi city, Guangxi province. The particle size of undisturbed soil evenly distributes $(2.43 \mathrm{~mm})$ and takes a percentage of over $80 \%$. The particle size of contaminated soil is $7.15 \mathrm{~mm}$ and takes a percentage of over $55 \%$. There is a significant difference in particle size between undisturbed soil and contaminated soil. Spectrum detector (SSFD-600) was used to determine the heavy metals, and the main heavy metals mainly contain lead, zinc and cadmium, the content of which is $0.15 \%, 0.42 \%$ and $1.01 \%$ respectively. The average comprehensive content is $0.53 \%$. According to the determination results of the physical property of soil samples, the water content of most contaminated soil samples is below the liquid limit, which belongs to low liquid limit type soil. The main physical property parameters of soil samples can be seen in Table 1.

Table 1. Main physical properties of soil samples.

\begin{tabular}{|c|c|c|c|c|c|c|c|c|c|}
\hline $\begin{array}{c}\text { Sample } \\
\text { No. }\end{array}$ & $\begin{array}{c}\text { Water } \\
\text { content } \omega /\end{array}$ & Density $\rho$ / & Porosity $e$ / & $\begin{array}{c}\text { Liquid limit } \\
w_{\mathrm{L}} / \\
0\end{array}$ & $\begin{array}{c}\text { Plastic limit } \\
w_{\mathrm{p}} / \\
0\end{array}$ & $\begin{array}{l}\text { Plasticity } \\
\text { index } I_{\mathrm{P}} /\end{array}$ & $\begin{array}{l}\text { Liquidity } \\
\text { index } I_{\mathrm{L}} /\end{array}$ & $\begin{array}{c}\text { Free swelling } \\
\text { ratio } F_{\mathrm{s}} /\end{array}$ & $\begin{array}{c}\text { Swelling without } \\
\text { load } V_{\mathrm{H}} /\end{array}$ \\
\hline & $\%$ & $\mathrm{~g} / \mathrm{cm}^{3}$ & & & & & & & \\
\hline 1 & 13.85 & 20.16 & 0.87 & $33.0 \sim 40.0$ & 15.72 & 20.34 & 0.19 & 23.6 & 11.95 \\
\hline 2 & 10.89 & 19.22 & 0.79 & 34.5 & 15.34 & 21.27 & 0.26 & 22.7 & 6.78 \\
\hline 3 & 12.35 & 20.34 & 0.83 & 35.2 & 16.29 & 22.35 & 0.28 & 26.9 & 9.57 \\
\hline 4 & 14.65 & 19.42 & 0.91 & 35.7 & 17.27 & 17.42 & 0.19 & 28.5 & 11.78 \\
\hline
\end{tabular}




\begin{tabular}{|c|c|c|c|c|c|c|c|c|c|}
\hline 5 & 16.9 & 19.17 & 0.75 & 34.8 & 17.35 & 16.98 & 0.27 & 24.9 & 9.65 \\
\hline 6 & 12.52 & 19.69 & 0.78 & 34.1 & 16.42 & 21.73 & 0.19 & 22.7 & 7.92 \\
\hline 7 & 11.77 & 19.11 & 0.81 & 35.7 & 15.98 & 17.42 & 0.24 & 29.5 & 8.56 \\
\hline 8 & 16.45 & 20.76 & 0.85 & 36.8 & 16.73 & 19.13 & 0.28 & 26.2 & 9.57 \\
\hline 9 & 14.51 & 19.28 & 0.74 & 37.9 & 17.42 & 21.72 & 0.21 & 27.9 & 11.89 \\
\hline 10 & 12.37 & 20.34 & 0.83 & 35.2 & 16.29 & 22.35 & 0.28 & 26.9 & 9.57 \\
\hline 11 & 14.65 & 19.42 & 0.91 & 35.7 & 17.27 & 17.42 & 0.19 & 28.5 & 11.78 \\
\hline 12 & 16.9 & 19.17 & 0.75 & 34.8 & 17.35 & 16.98 & 0.27 & 24.8 & 9.64 \\
\hline 13 & 12.52 & 19.69 & 0.78 & 34.1 & 16.42 & 21.73 & 0.19 & 22.7 & 7.92 \\
\hline 14 & 12.31 & 20.34 & 0.83 & 35.2 & 16.29 & 22.35 & 0.28 & 26.9 & 9.57 \\
\hline 15 & 12.33 & 20.37 & 0.88 & 35.1 & 16.27 & 22.31 & 0.26 & 26.1 & 9.53 \\
\hline 16 & 12.78 & 20.39 & 0.89 & 38.3 & 17.13 & 22.23 & 0.26 & 28.6 & 12.16 \\
\hline 17 & 10.62 & 19.41 & 0.92 & 36.9 & 16.72 & 17.58 & 0.29 & 23.7 & 9.45 \\
\hline 18 & 12.79 & 19.36 & 0.75 & 36.2 & 17.23 & 21.27 & 0.22 & 27.9 & 8.76 \\
\hline 19 & 12.39 & 20.34 & 0.83 & 35.2 & 16.29 & 22.35 & 0.28 & 26.9 & 9.57 \\
\hline 20 & 14.65 & 19.42 & 0.91 & 35.7 & 17.27 & 17.42 & 0.19 & 28.5 & 11.78 \\
\hline
\end{tabular}

\subsection{Soil samples preparation}

The undisturbed soil was used to prepare three types of soils, i.e., undisturbed soil, contaminated soil, and contaminated soil solidified with the organic curing agent (abbreviated it as solidified soil). Each soil sample has 20 replicates. The preparation of undisturbed soil and contaminated soil was done according to the conventional soil sample preparation method, and the preparation of solidified soil was done according to the mix proportion scheme of organic agents (seen in Table 2).

Table 2. Mix proportion scheme of soil samples.

\begin{tabular}{|c|c|c|c|c|c|c|c|}
\hline \multirow[t]{2}{*}{ No. } & \multicolumn{4}{|c|}{ Solidified soil } & \multirow{2}{*}{\begin{tabular}{|c|} 
Undisturbed soil \\
$\begin{array}{c}\text { Water content } \\
/ \%\end{array}$ \\
\end{tabular}} & \multicolumn{2}{|c|}{ Contaminated soil } \\
\hline & $\begin{array}{c}\text { Epoxy agent } \\
/ \%\end{array}$ & $\begin{array}{c}\text { Epoxy accelerant } \\
/ \%\end{array}$ & Diluent/\% & Water content $/ \%$ & & $\begin{array}{c}\text { Contamination content } \\
/ \%\end{array}$ & Water content $\omega / \%$ \\
\hline 1 & 1.58 & 1.81 & 2.12 & 13.24 & 13.69 & 3.125 & 13.56 \\
\hline 2 & 2. 19 & 2.01 & 2.14 & 13.54 & 13.53 & 4. 214 & 13.68 \\
\hline 3 & 1.56 & 2.64 & 2. 16 & 13.56 & 13.68 & 3.662 & 13.53 \\
\hline 4 & 1.59 & 1.83 & 2. 36 & 13.68 & 13.25 & 6. 743 & 13.25 \\
\hline 5 & 2. 16 & 2.53 & 2.20 & 13.53 & 13.19 & 6.514 & 13.69 \\
\hline 6 & 1.51 & 2.64 & 2.22 & 13.25 & 13.69 & 4. 217 & 13.53 \\
\hline 7 & 1.52 & 1.85 & 2.24 & 13.69 & 13.47 & 5. 185 & 13.68 \\
\hline 8 & 2. 16 & 2.64 & 2.26 & 13.53 & 13.72 & 4. 323 & 13.25 \\
\hline 9 & 1.52 & 2.72 & 2.45 & 13. 34 & 13.68 & 6. 107 & 13. 19 \\
\hline 10 & 1.57 & 1.88 & 2.32 & 13.17 & 13.25 & 5.325 & 13.69 \\
\hline 11 & 2. 18 & 2. 66 & 2.56 & 13.68 & 13.19 & 3. 977 & 13.47 \\
\hline 12 & 1. 49 & 1.86 & 2.32 & 13.25 & 13.69 & 7. 186 & 13.72 \\
\hline 13 & 2.27 & 2.69 & 2. 19 & 13.19 & 13.47 & 5. 342 & 13.68 \\
\hline 14 & 1. 51 & 2. 12 & 2. 17 & 13.69 & 13.53 & 9.283 & 13.25 \\
\hline 15 & 1. 52 & 1.95 & 2.32 & 13.47 & 13.25 & 8. 194 & 13.19 \\
\hline 16 & 2.17 & 2.71 & 2. 19 & 13.72 & 13.69 & 4. 323 & 13.69 \\
\hline 17 & 1. 56 & 2.64 & 2.32 & 13.64 & 13.53 & 3. 107 & 13.47 \\
\hline 18 & 1. 53 & 1.92 & 2.37 & 13.43 & 13.34 & 4. 325 & 13.72 \\
\hline 19 & 2.13 & 2.73 & 2.13 & 13.25 & 13.17 & 3.977 & 13.64 \\
\hline 20 & 2. 24 & 2.83 & 2. 19 & 13.14 & 13.53 & 10.467 & 13.68 \\
\hline
\end{tabular}

The undisturbed soil and contaminated soil samples were dried, grounded, and screened through $2 \mathrm{~mm}$ sieve and then set them as the experiment samples. According to the mix proportion scheme of Table 2, soil and water were accurately weighed and mixed evenly, and then poured and vibrated in the trial mold to complete the preparation of experimental soil samples. According to the mix proportion scheme of solidified soil in Table 2, the contaminated soil, curing agent and water were accurately weighed. Curing agent and water were mixed and vortexed and poured into soil, and balled it up in the trial mold. The finished samples were placed in a humidifier for 24 hours for further use.

\subsection{Consolidated drained triaxial shear test (CD)}

The size of the samples is standard, diameter $3.91 \mathrm{~cm}$, height $8.0 \mathrm{~cm}$. In consideration of the wide variance on characteristic change of dilatancy and contraction of contaminated soil, two sorts of confining pressures were involved in, i.e., low confining, 90, 120, 240kPa, and medium to high confining, 600, 900, 1200kPa. The saturated drained triaxial shear test was done under each of the confining pressure, and the results were analyzed and compared to investigate the changes of mechanical strength and dilatancy (contraction) characteristics of heavy metal contaminated soil before and after consolidation. 


\subsection{Results and discussion}

In Figure 2-7, relationship curves of $q-\varepsilon_{1}, \varepsilon_{\mathrm{v}}-\varepsilon_{1}$ pressure and medium to high confining pressure are shown using consolidated drained triaxial shear test, respectively. and $\sigma_{1} / \sigma_{3}-\varepsilon_{1}$ of samples under low confining
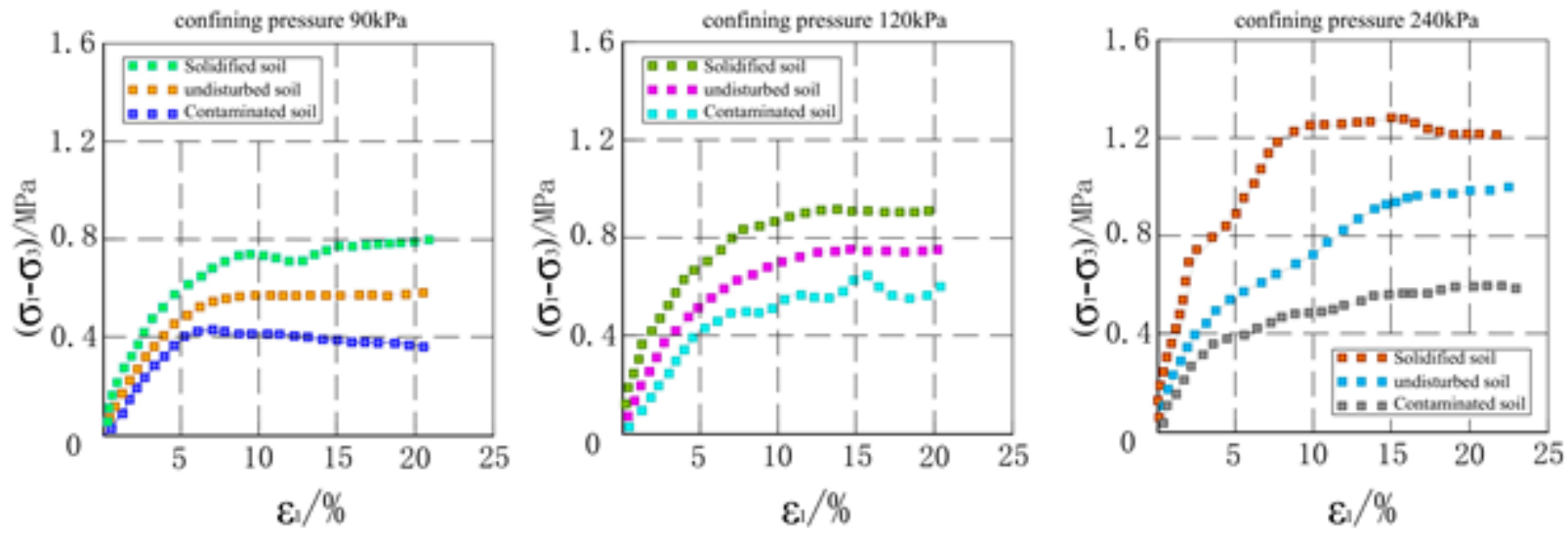

Fig. 2. $q-\varepsilon_{1}$ curve comparison under low confining pressure
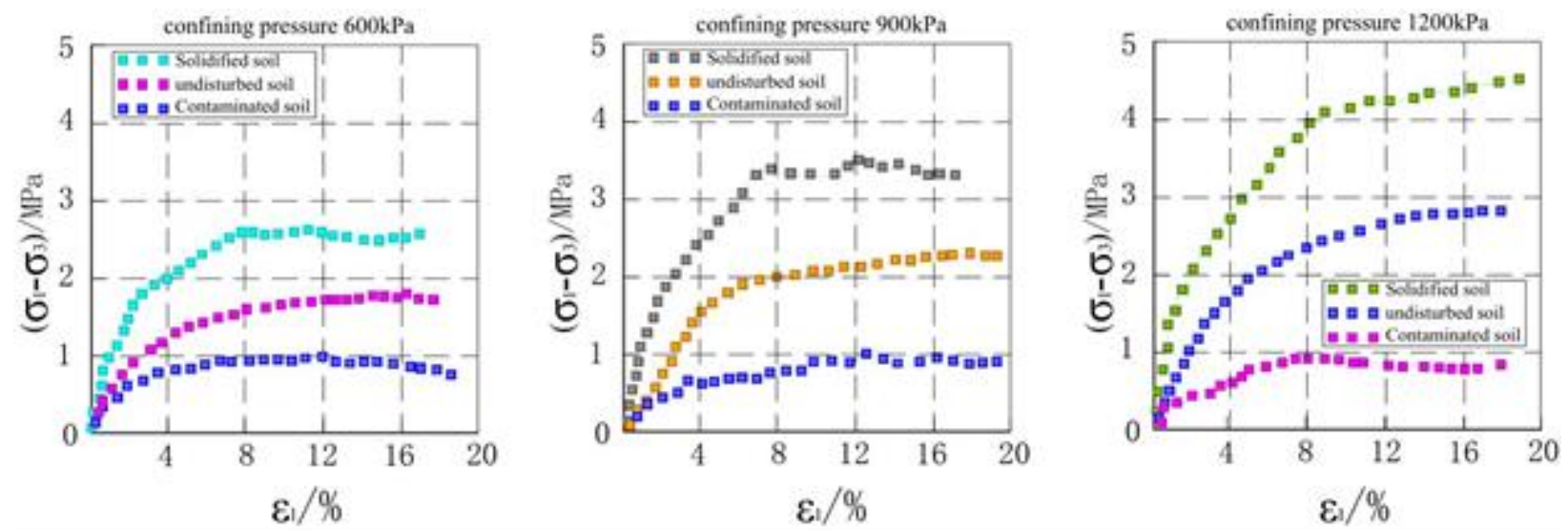

Fig. 3. $q-\varepsilon_{1}$ curve comparison under medium to high confining pressure.

Seen from Figure 2 and Figure 3, under low confining pressure and medium to high confining pressure, peak strength of undisturbed soil, contaminated soil, and solidified soil appear different change rule, among which the peak strength increment of the solidified soil is the most significant. With the increase of confining pressure, the peak strength increment of contaminated soil is far lower than undisturbed soil and solidified soil, while after adding the curing agent, the peak strength of contaminated soil was improved by nearly $35 \%$ and $55 \%$ under low confining pressure and high confining pressure, respectively. Even the increment is higher than undisturbed soil, which indicates that the curing agent plays a great role in improving the strength of soil mass.
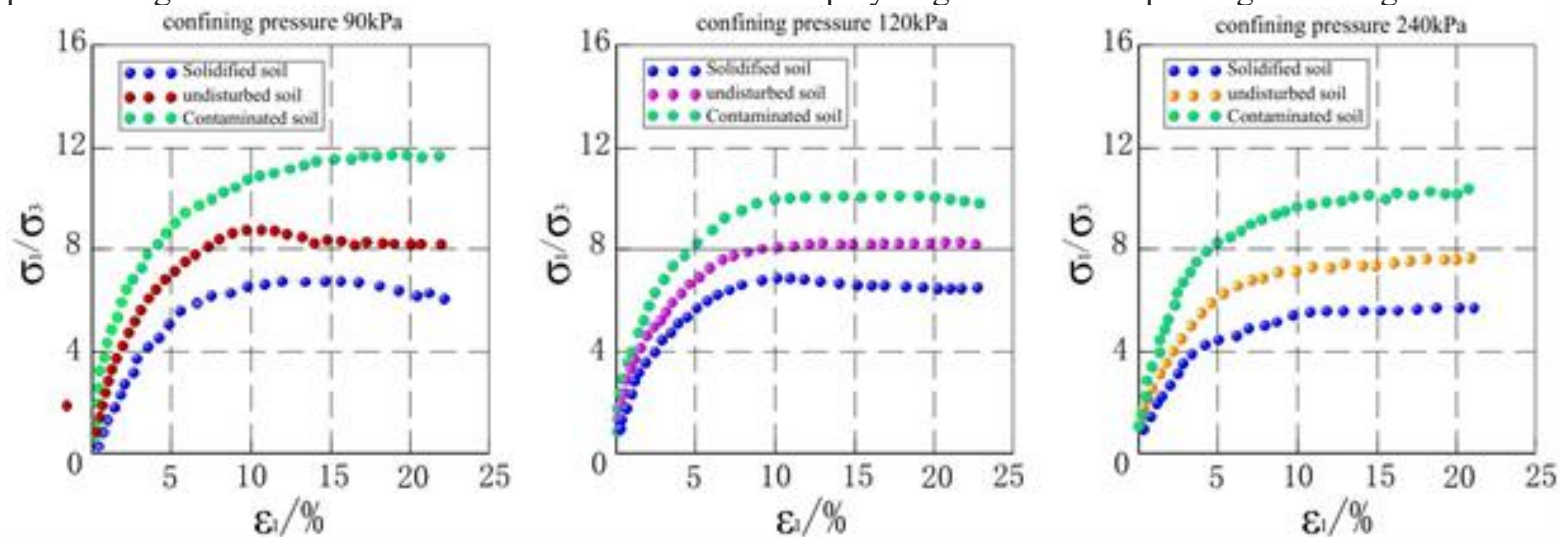

Fig. 4. $\sigma_{1} / \sigma_{3}-\varepsilon_{1}$ curve comparison under low confining pressure. 

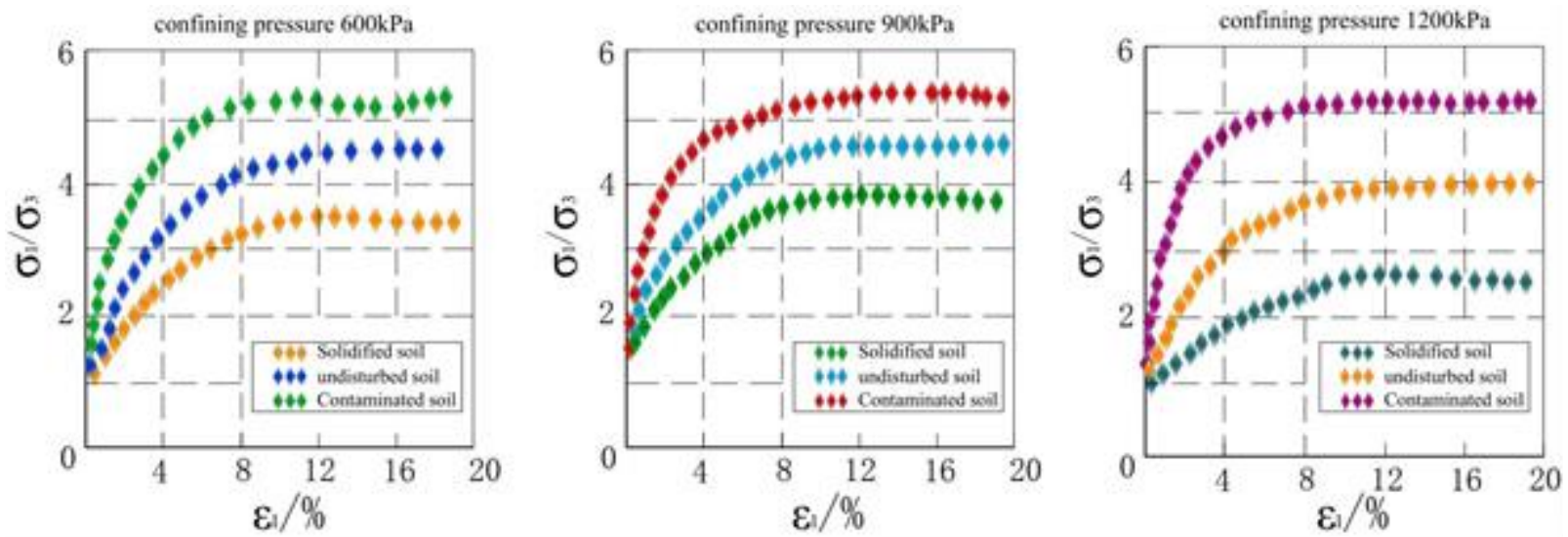

Fig. 5. $\sigma_{1} / \sigma_{3}-\varepsilon_{1}$ curve comparison under medium to high confining pressure.

Seen from Figure 4 and Figure 5, the samples of contaminated soil display high peak principle stress ration under low, medium, and high confining pressure, which indicates that the anti-disturbance capacity of soil mass has weak disturbance resistance, poor stability and low shear resistance. While the peak principal stress of solidified soil is stable and relatively lower under different confining pressure, which indicates that the antidisturbance ability of the soil after solidification is enhanced, the stability is improved, and the stability is better than that of the undisturbed soil.
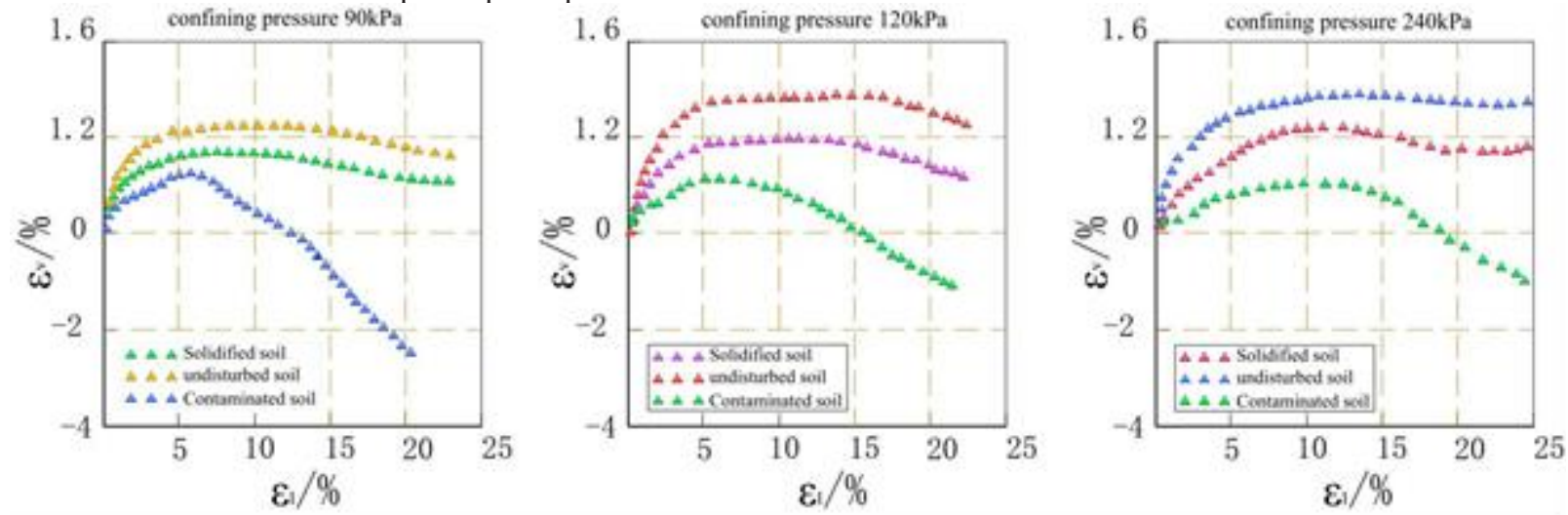

Fig. 6. $\varepsilon_{\mathrm{v}}-\varepsilon_{1}$ curve result comparison under low confining pressure.
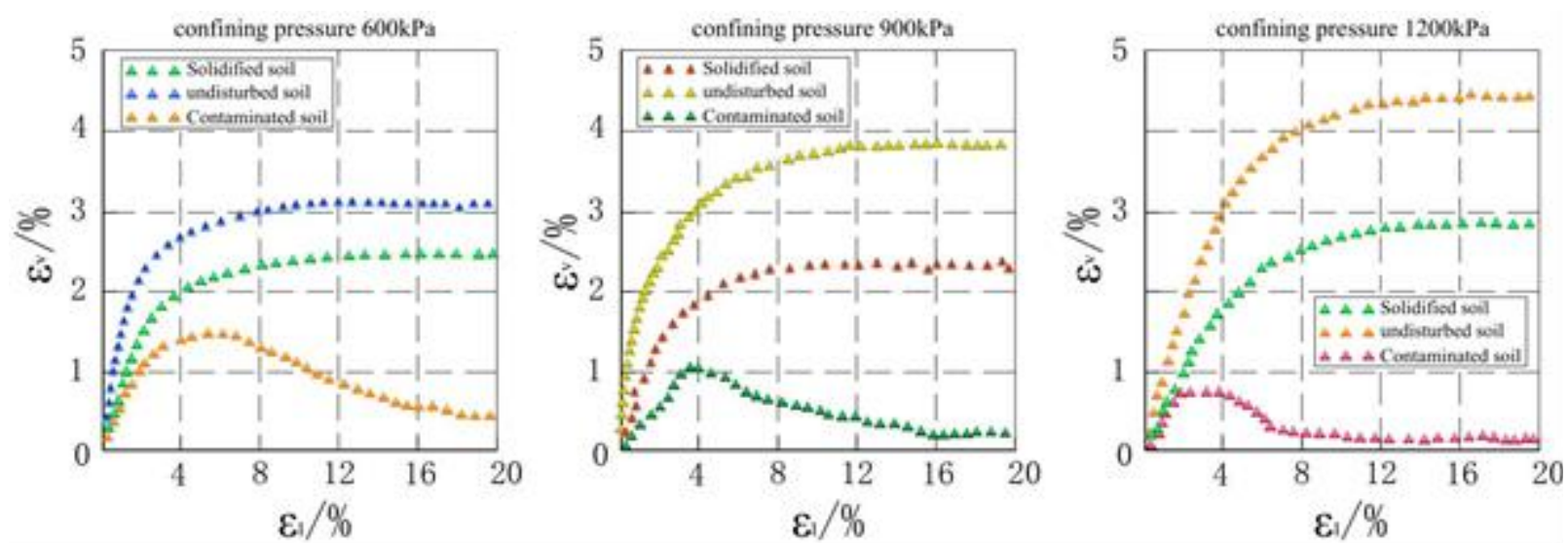

Fig. 7. $\varepsilon_{\mathrm{v}}-\varepsilon_{1}$ curve result comparison under medium to high confining pressure.

Figure 6 and Figure 7 show that the contaminated soil sample appears strain softened feature after peak shear stress, while the solidified soil sample appears strain hardened feature after peak shear stress. Through comparing the $\varepsilon_{\mathrm{v}}-\varepsilon_{1}$ relationship curves of the two soil samples, the contaminated soil sample underwent contraction $\left(\varepsilon_{\mathrm{v}}>0\right)$ first and then significant dilatancy $\left(\varepsilon_{\mathrm{v}}<0\right)$ under the condition of low confining pressure. Under medium to high confining pressure, dilatancy is 
gradually impaired, and contraction is enhanced gradually. For solidified soil sample, swell-shrinking deformation is relatively stable. Within the strain range, the contracted deformation is constantly observed instead of dilatancy, except that undisturbed soil appeared dilatancy at critical shear failure, which is basically consistent with the swellshrinking property of undisturbed soil. It indicates that after being invaded by heavy metals the internal structure of undisturbed soil is destroyed to a large extent, the adhesive force is impaired, and the deformation behavior is weakened. Once undisturbed soil is acted by an external force, it will largely deform which influences its function. Solidified soil shows excellent volumetric strain characteristics after shear deformation, which sufficiently indicates that the organic curing agents has better effects on improving volumetric dilatancy (contraction) characteristics of soil mass.

\section{Volumetric strain characteristics constitutive model}

Adhesion and migration of heavy metal in soil mass leads to the configuration change and dilatancy (contraction) of soil mass ${ }^{[8,9]}$, among which the main plastic strain deformation is the displacement and sliding of the particles $^{[10]}$, especially when loaded, and mechanical property is like the coarse-grained soil ${ }^{[11]}$. In order to reflect this volumetric strain characteristics a function that is suitable to coarse-grained soil was based on in this article. The double yield surface constitutive model of heavy metal contaminated soil is established by combining the shear yield function and the compression yield function.

\subsection{Initial function}

\subsubsection{Shear yield function}

Obtained from results mentioned above, shearing force properties of heavy metal contaminated soil mainly are contraction and dilatancy, and its shear principle fits in Pietruszczak rule ${ }^{[9]}$, which is

$$
M=\frac{\varepsilon_{\mathrm{s}}^{\mathrm{p}}}{B+\varepsilon_{\mathrm{s}}^{\mathrm{p}}} M_{\mathrm{p}}
$$

where $\mathrm{B}$ is material constant, $\varepsilon_{\mathrm{s}}^{\mathrm{p}}$ is shear strain, and $M_{\mathrm{p}}$ is peak value stress ratio.

Three-dimensional generalization of Gajo and $\mathrm{Wood}^{[9,}$ ${ }^{10]}$ was used as the specific form of the shear equation:

$$
D_{1}=A\left[M_{\mathrm{d}} g(\theta)-\eta\right]
$$

where $A$ is model parameter, $M_{\mathrm{d}}$ is transformed stress ratio representing $p-q$ relationship curve transformed slope, $p$ is average positive stress, $q$ is shear stress, $q=\sigma_{1}-\sigma_{3}, g(\theta)$ is shape function, $\theta$ is stress position angle, $\theta=\pi / 6$, and $\eta$ is stress ratio, $\eta=q / p$.
According to the stress state transformation conception of Ishihara et al. ${ }^{[9]}$, the relationship of residual stress ratio $M_{\mathrm{r}}$, peak value stress ration $M_{\mathrm{P}}$ and the void ration is as following:

$$
\frac{M-M_{\mathrm{P}}}{M_{\mathrm{r}}-M_{\mathrm{P}}}=1-\mathrm{e}^{(-\mathrm{aR})}
$$

where $M_{\mathrm{r}}$ is residual stress ratio, $M_{\mathrm{p}}$ is peak value stress ratio, $e$ is void ratio, $a$ is model parameter, and $R$ is shape parameter.

According to the definition of dilatancy ${ }^{[10,12]}$, the ratio of volumetric strain increment $\varepsilon_{\mathrm{v}}^{\mathrm{p}}$ to shear strain increment represents dilatancy of soil mass, and its equation is

$$
D_{1}=d \varepsilon_{\mathrm{v}}^{\mathrm{p}} / d \varepsilon_{\mathrm{s}}^{\mathrm{p}}
$$

After collation of equation (1) - (4), the equivalent form of shear yield function is

$$
g_{1}=q+\frac{A}{1-A} M_{\mathrm{d}} p g(\theta)\left[1-\left(\frac{p}{p_{0}}\right)^{-(1-A)}\right]=0
$$

where $p_{0}$ is initial effective average stress.

For the convenience of calculation, equation (5) was expressed using the simplified form of Stolle's double yield surface curve, it is as following:

$$
f_{1}=q-\operatorname{Mg}(\theta) p=0
$$

where $M$ is residual stress ratio, $g(\theta)$ is shape function and its expression is

$$
g(\theta)=\frac{3-\sin \varphi}{(3-\sin \varphi)+\sqrt{2} \sin \varphi \sqrt{1-\sin (3 \theta)}}
$$

where $\varphi$ is internal friction angle.

\subsubsection{Compression yield function}

The compression property of heavy metal contaminated soil is almost the same as undisturbed soil. For the pure compressive deformation mechanism, the corrected Cambridge model form, in combination with associative flow rule, can better reflect volumetric strain aroused by compression $^{[8,}{ }^{10-12]}$. Through introducing the shape parameter $R$, the specific yield function and plastic function forms are as following:

$$
\begin{aligned}
f_{2}=g_{2}= & \left(\frac{p}{p_{c}}\right)^{2}+(R-1)^{2}\left(\frac{q}{M p_{c}}\right)^{2}- \\
& \frac{2}{R} \frac{p}{p_{c}}+\frac{2-R}{R}=0
\end{aligned}
$$

where $p_{c}$ is solidified pressure.

For shear deformation, heavy metal contaminated soil shows obvious isotropic hardening, and its volumetric strain process can be expressed using the corrected Cambridge model as follow. 


$$
d \varepsilon_{\mathrm{v}}^{\mathrm{p}}=\left(\lambda^{*}-\kappa^{*}\right) \frac{d p_{c}}{p_{c}}
$$

where $d p_{c}$ is solidified pressure increment, $\lambda^{*}$ is slope of $v-\ln p$ compressive curve, $v$ is specific volume, and $\kappa^{*}$ is slope of $v-\ln p$ rebound curve.

\subsection{Hyperboloid constitutive equation}

According to the above definition of the initial function, the explicit numerical integration algorithm was used, and the volumetric strain and shear strain were expressed as follows.

$$
\begin{aligned}
& \mathrm{d} \varepsilon_{\mathrm{v}}^{\mathrm{p}}=\mathrm{d} \lambda_{1} \frac{\partial g_{1}}{\partial p}+\mathrm{d} \lambda_{2} \frac{\partial g_{2}}{\partial p} \\
& \mathrm{~d} \varepsilon_{\mathrm{s}}^{\mathrm{p}}=\mathrm{d} \lambda_{1} \frac{\partial g_{1}}{\partial q}+\mathrm{d} \lambda_{2} \frac{\partial g_{2}}{\partial q}
\end{aligned}
$$

where $\lambda_{1}$ is compressive curve slope, and $\lambda_{2}$ is shear curve slope.

by $\mathrm{d} f_{1}=0$,

$$
\mathrm{d} \lambda_{1}=\frac{1}{H_{\mathrm{p} 1}}\left(\frac{\partial f_{1}}{\partial p} d p+\frac{\partial f_{1}}{\partial q} d q\right)
$$

where $H_{\mathrm{p} 1}=-\frac{\partial f_{1}}{\partial M} \frac{\partial M}{\partial \varepsilon_{\mathrm{s}}^{\mathrm{p}}} \frac{\partial g_{1}}{\partial q}$.

by $\mathrm{d} f_{2}=0$,

$$
\mathrm{d} \lambda_{2}=\frac{1}{H_{\mathrm{p} 2}}\left(\frac{\partial f_{2}}{\partial p} \mathrm{~d} p+\frac{\partial f_{2}}{\partial q} \mathrm{~d} q\right)
$$

where $H_{\mathrm{p} 2}=-\frac{\partial f_{2}}{\partial p_{\mathrm{c}}} \frac{\partial p_{\mathrm{c}}}{\partial \varepsilon_{\mathrm{v}}^{\mathrm{p}}} \frac{\partial g_{2}}{\partial p}$.

Substitute (12) and (13) in equation (10) and (11),

$$
\begin{aligned}
\mathrm{d} \varepsilon_{\mathrm{v}}^{\mathrm{p}}=\frac{1}{H_{\mathrm{p} 1}}\left(\frac{\partial f_{1}}{\partial p} \frac{\partial g_{1}}{\partial p} \mathrm{~d} p+\frac{\partial f_{1}}{\partial q} \frac{\partial g_{1}}{\partial p} \mathrm{~d} q\right)+ \\
\frac{1}{H_{\mathrm{p} 2}}\left(\frac{\partial f_{2}}{\partial p} \frac{\partial g_{2}}{\partial p} \mathrm{~d} p+\frac{\partial f_{2}}{\partial q} \frac{\partial g_{2}}{\partial p} \mathrm{~d} q\right)
\end{aligned}
$$

$$
\begin{aligned}
\mathrm{d} \varepsilon_{\mathrm{s}}^{\mathrm{p}}=\frac{1}{H_{\mathrm{p} 1}}\left(\frac{\partial f_{1}}{\partial p} \frac{\partial g_{1}}{\partial q} d p+\frac{\partial f_{2}}{\partial q} \frac{\partial g_{1}}{\partial q} \mathrm{~d} q\right)+ \\
\frac{1}{H_{\mathrm{p} 2}}\left(\frac{\partial f_{2}}{\partial p} \frac{\partial g_{2}}{\partial q} \mathrm{~d} p+\frac{\partial f_{2}}{\partial q} \frac{\partial g_{2}}{\partial q} \mathrm{~d} q\right) \\
\mathrm{d} p=K\left(\mathrm{~d} \varepsilon_{\mathrm{v}}-\varepsilon_{\mathrm{v}}^{\mathrm{p}}\right) \\
\mathrm{d} q=3 G\left(\mathrm{~d} \varepsilon_{\mathrm{s}}-\varepsilon_{\mathrm{s}}^{\mathrm{p}}\right)
\end{aligned}
$$

where $K$ is slope coefficient of curve, and $G$ is material constant.

$$
\begin{aligned}
\mathrm{d} \varepsilon_{\mathrm{v}}= & \frac{1}{K} \mathrm{~d} p+\frac{1}{H_{\mathrm{p} 1}}\left(\frac{\partial f_{1}}{\partial p} \frac{\partial g_{1}}{\partial p} \mathrm{~d} p+\frac{\partial f_{1}}{\partial q} \frac{\partial g_{1}}{\partial p} \mathrm{~d} q\right)+ \\
& \frac{1}{H_{\mathrm{p} 2}}\left(\frac{\partial f_{2}}{\partial p} \frac{\partial g_{2}}{\partial p} \mathrm{~d} p+\frac{\partial f_{2}}{\partial q} \frac{\partial g_{2}}{\partial p} \mathrm{~d} q\right) \\
\mathrm{d} \varepsilon_{\mathrm{s}}= & \frac{1}{3 G} \mathrm{~d} q+\frac{1}{H_{\mathrm{p} 1}}\left(\frac{\partial f_{1}}{\partial p} \frac{\partial g_{1}}{\partial q} \mathrm{~d} p+\frac{\partial f_{2}}{\partial q} \frac{\partial g_{1}}{\partial q} \mathrm{~d} q\right)+ \\
& \frac{1}{H_{\mathrm{p} 2}}\left(\frac{\partial f_{2}}{\partial p} \frac{\partial g_{2}}{\partial q} \mathrm{~d} p+\frac{\partial f_{2}}{\partial q} \frac{\partial g_{2}}{\partial q} \mathrm{~d} q\right)
\end{aligned}
$$

or

$$
\left\{\begin{array}{l}
a_{11} \mathrm{~d} p+a_{12} \mathrm{~d} q=\mathrm{d} \varepsilon_{\mathrm{v}} \\
a_{21} \mathrm{~d} p+a_{22} \mathrm{~d} q=\mathrm{d} \varepsilon_{\mathrm{s}}
\end{array}\right.
$$

where $a_{11}=\frac{1}{K}+\frac{1}{H_{\mathrm{p} 1}} \frac{\partial f_{1}}{\partial p} \frac{\partial g_{1}}{\partial p}+\frac{1}{H_{\mathrm{p} 2}} \frac{\partial f_{2}}{\partial p} \frac{\partial g_{2}}{\partial p}$,

$a_{12}=\frac{1}{H_{\mathrm{p} 1}} \frac{\partial f_{1}}{\partial q} \frac{\partial g_{1}}{\partial p}+\frac{1}{H_{\mathrm{p} 2}} \frac{\partial f_{2}}{\partial q} \frac{\partial g_{2}}{\partial p}$,

$a_{21}=\frac{1}{H_{\mathrm{p} 1}} \frac{\partial f_{1}}{\partial p} \frac{\partial g_{1}}{\partial q}+\frac{1}{H_{\mathrm{p} 2}} \frac{\partial f_{2}}{\partial p} \frac{\partial g_{2}}{\partial q}$,

$a_{22}=\frac{1}{3 G}+\frac{1}{H_{\mathrm{p} 1}} \frac{\partial f_{1}}{\partial q} \frac{\partial g_{1}}{\partial q}+\frac{1}{H_{\mathrm{p} 2}} \frac{\partial f_{2}}{\partial q} \frac{\partial g_{2}}{\partial p}$.

Normally, the confining pressure of the drained triaxial strain test is controlled unchanged ${ }^{[15]}$, that means $\mathrm{d} \sigma_{3}=0, \mathrm{~d} \varepsilon_{1}$ and $\mathrm{d} \sigma_{3}$ are known. For this condition, it is derived as follows

$$
\begin{aligned}
& 3 \mathrm{~d} \varepsilon_{1}=\mathrm{d} \varepsilon_{\mathrm{v}}+3 \mathrm{~d} \varepsilon_{\mathrm{s}} \\
& 3 \mathrm{~d} \sigma_{3}=3 \mathrm{~d} p-\mathrm{d} q
\end{aligned}
$$

where $\varepsilon_{1}$ is principal strain, $\varepsilon_{\mathrm{v}}$ is contraction strain, and $\varepsilon_{\mathrm{s}}$ is dilatancy strain.

Substitute (19) in equation (20) and combined with (21)

$$
\left\{\begin{array}{l}
a_{11}^{*} \mathrm{~d} p+a_{12}^{*} \mathrm{~d} q=b_{1}^{*} \\
a_{21}^{*} \mathrm{~d} p+a_{22}^{*} \mathrm{~d} q=b_{2}^{*}
\end{array}\right.
$$

where $a_{11}^{*}=a_{11}+3 a_{21}, \quad a_{12}^{*}=a_{12}+3 a_{22}, \quad a_{21}^{*}=3$, $a_{22}^{*}=-1, \quad b_{1}^{*}=3 \mathrm{~d} \varepsilon_{1}, \quad b_{2}^{*}=3 \mathrm{~d} \sigma_{3}$.

Equation (23) was numerically integrated using procedure Matlab, and $\mathrm{d} p$ and $\mathrm{d} q$ were obtained, and then substitute them in equation (20) to obtain $\mathrm{d} \varepsilon_{\mathrm{v}}$ and $\mathrm{d} \varepsilon_{\mathrm{s}}$.

\subsection{Determination of model parameters}

The model has a total of 10 material parameters from the experimental result curve of the abovementioned contaminated soil. $A$ is dilatancy parameter, $h_{\mathrm{v}}$ and 
$m$ are hardening parameters determined by $\varepsilon_{\mathrm{v}}-\varepsilon_{1}$ curve; $\varepsilon_{\mathrm{s}, \mathrm{d}}^{\mathrm{p}}$ is plastic shear strain determined by $\varepsilon_{\mathrm{s}}^{\mathrm{p}}-\eta$ curve; $B$ and $a$ are softening parameters determined by $q-\varepsilon_{1}$ curve; $G_{0}$ is material parameter determined by $q-\varepsilon_{\mathrm{s}}$ of triaxial test unloaded; $M_{\mathrm{p}}$ is the corresponding $\eta=q / p$ peak shear strength; $M_{\mathrm{r}}$ is residual state stress ratio determined by $\eta-\varepsilon_{1}$ curve; $v$ is volume ratio determined by $p-\varepsilon_{\mathrm{v}}$ curve when unloaded by equation $K=G 2(1+v) /[3(1-2 v)]$. According to results of reference ${ }^{[3]},{ }^{[12-16]}$, three groups of data were applied to determine the parameters of model (see Table 3).

Table 3. Calculation parameters of the model.

\begin{tabular}{|c|c|c|c|c|c|c|c|c|c|c|}
\hline $\begin{array}{c}\text { Sample } \\
\text { No. }\end{array}$ & $\begin{array}{c}\text { Dilatancy } \\
\text { parameter } \\
A \\
\end{array}$ & $\begin{array}{c}\text { Softening } \\
\text { parameter } \\
B \\
\end{array}$ & $\begin{array}{c}\text { Plastic shear } \\
\text { strain } \\
\varepsilon_{\mathrm{s}, \mathrm{d}}^{\mathrm{p}} \\
\end{array}$ & $\begin{array}{c}\text { Shear } \\
\text { strength } \\
M_{\mathrm{p}} \\
\end{array}$ & $\begin{array}{c}\text { Softening } \\
\text { parameter } \\
\quad a \\
\end{array}$ & $\begin{array}{c}\text { Residual state } \\
\text { stress ratio } \\
M_{\mathrm{r}} \\
\end{array}$ & $\begin{array}{c}\text { Hardening } \\
\text { parameter } \\
h_{\mathrm{v}} \\
\end{array}$ & $\begin{array}{c}\text { Hardening } \\
\text { parameter } \\
m \\
\end{array}$ & $\begin{array}{c}\text { Material } \\
\text { parameters } \\
G_{0}\end{array}$ & $\begin{array}{c}\text { Specific } \\
\text { volume } \\
v \\
\end{array}$ \\
\hline 1 & 0.51 & 0.006 & 0.130 & 2. 184 & 4. 68 & 1. 49 & 0.49 & 0.81 & 430 & 0.26 \\
\hline 2 & 0.54 & 0.006 & 0.062 & 1. 681 & 4. 82 & 1. 52 & 0.54 & 0.86 & 420 & 0.19 \\
\hline 3 & 0.56 & 0.007 & 0.072 & 1. 924 & 4. 76 & 1.56 & 0.58 & 0.86 & 420 & 0.19 \\
\hline 4 & 0.51 & 0.006 & 0.129 & 2. 084 & 4.68 & 1.51 & 0.48 & 0.81 & 430 & 0.26 \\
\hline 5 & 0.54 & 0.006 & 0.064 & 1. 624 & 4.82 & 1.51 & 0.50 & 0.86 & 420 & 0.19 \\
\hline 6 & 0.55 & 0.007 & 0.076 & 1.931 & 4. 76 & 1.58 & 0.57 & 0.86 & 420 & 0.19 \\
\hline 7 & 0.51 & 0.006 & 0.133 & 2. 136 & 4.68 & 1. 49 & 0.49 & 0.81 & 430 & 0.26 \\
\hline 8 & 0.52 & 0.006 & 0.069 & 1. 612 & 4. 82 & 1.52 & 0.56 & 0.86 & 420 & 0.19 \\
\hline 9 & 0.55 & 0.007 & 0.078 & 1. 946 & 4. 76 & 1. 57 & 0.58 & 0.86 & 420 & 0.19 \\
\hline 10 & 0.51 & 0.006 & 0.132 & 2. 278 & 4. 68 & 1.54 & 0.48 & 0.81 & 430 & 0.26 \\
\hline 11 & 0.57 & 0.006 & 0.061 & 1. 639 & 4. 82 & 1.51 & 0.51 & 0.86 & 420 & 0.19 \\
\hline 12 & 0.56 & 0.007 & 0.072 & 1.924 & 4. 76 & 1.56 & 0.58 & 0.86 & 420 & 0.19 \\
\hline 13 & 0.51 & 0.006 & 0.129 & 2. 084 & 4. 68 & 1.51 & 0.48 & 0.81 & 430 & 0.26 \\
\hline 14 & 0.54 & 0.006 & 0.064 & 1. 624 & 4.82 & 1.51 & 0.50 & 0.86 & 420 & 0.19 \\
\hline 15 & 0.55 & 0.007 & 0.076 & 1.931 & 4. 76 & 1.58 & 0.57 & 0.86 & 420 & 0.19 \\
\hline 16 & 0.51 & 0.006 & 0.133 & 2. 136 & 4. 68 & 1. 49 & 0.49 & 0.81 & 430 & 0.26 \\
\hline 17 & 0.52 & 0.006 & 0.069 & 1. 612 & 4. 82 & 1.52 & 0.56 & 0.86 & 420 & 0.19 \\
\hline 18 & 0.55 & 0.007 & 0.078 & 1.946 & 4. 76 & 1.57 & 0.58 & 0.86 & 420 & 0.19 \\
\hline 19 & 0.56 & 0.007 & 0.072 & 1. 924 & 4. 76 & 1.56 & 0.58 & 0.86 & 420 & 0.19 \\
\hline 20 & 0.51 & 0.006 & 0.133 & 2. 136 & 4. 68 & 1.55 & 0.57 & 0.81 & 430 & 0.25 \\
\hline
\end{tabular}

\subsection{Model fitting results and discussion}

The relation between $q-\varepsilon_{1}, \quad \varepsilon_{\mathrm{v}}-\varepsilon_{1}$ and $\sigma_{1} / \sigma_{3}-\varepsilon_{1}$ were curved according to the abovementioned 3 groups of samples. The parameters in Table 3 were treated as calculating parameters in double yield surface strain softened elastoplastic constitutive model, calculated by using Matlab and curved the fitting line. Comparison curves of experimental results and model fitting results are shown in Figure 8 and Figure 9
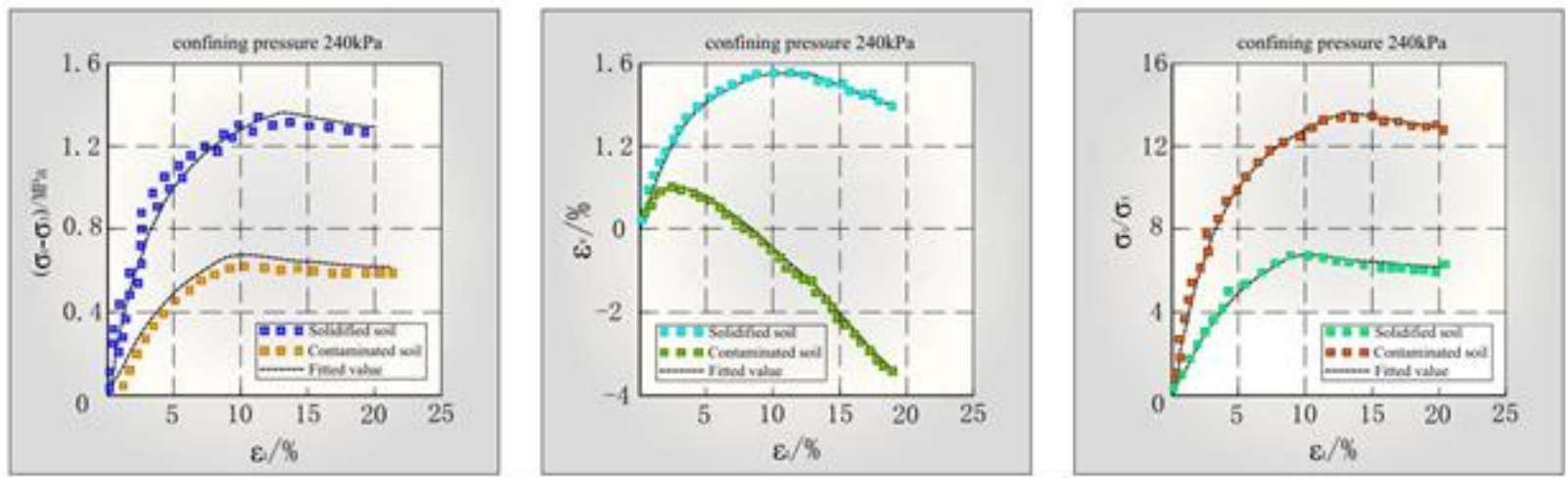

Fig. 8. Comparison of experimental results and model fitting results under low confining pressure. 

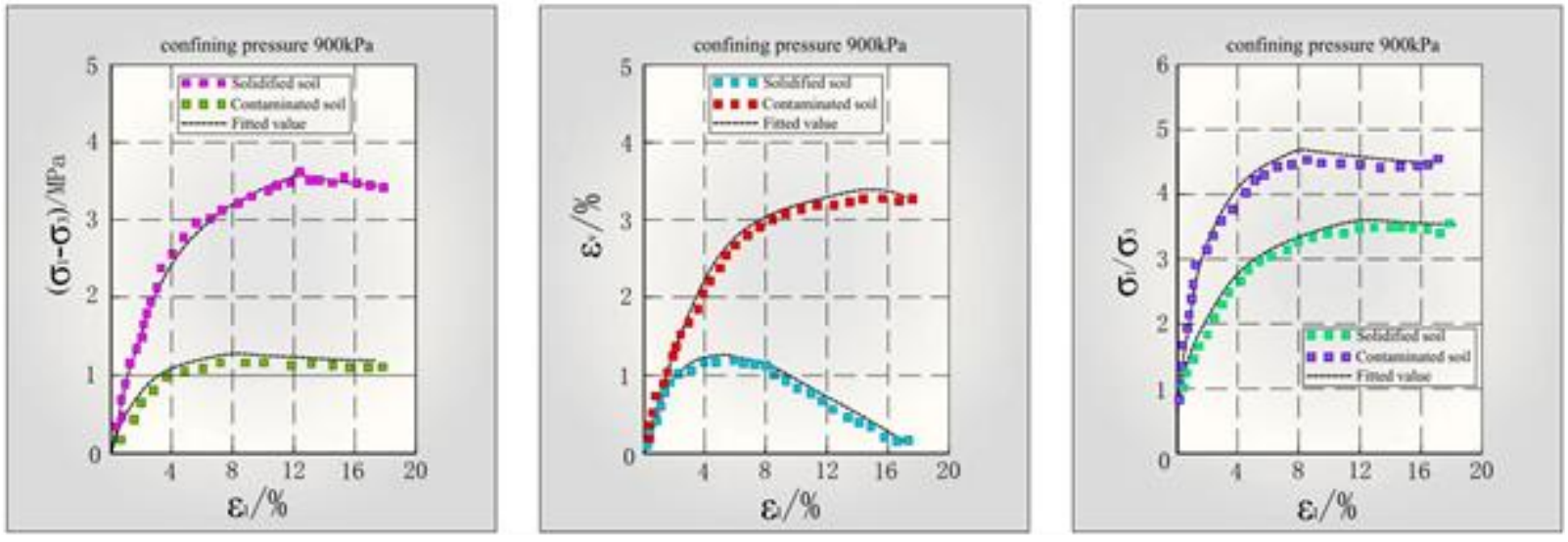

Fig. 9. Comparison of experimental results and model fitting results under medium to high confining pressure.

The fitting curves in Figure 8 and Figure 9 show that the model fitting results are in good agreement with the test results, which can better reflect the stress level change of heavy metal contaminated soil under the two confining pressures and the volumetric strain change process from contraction to dilatancy. The model fitting results further verify that the organic curing agent can effectively improve the shear strength of heavy metal contaminated soil, and improve the deformation performance of the soil under low confining pressure and high confining pressure.

\section{Conclusions}

1) The organic curing agents can significantly improve the peak strength of heavy metal contaminated soil. The peak strength can be increased by nearly $35 \% \sim 55 \%$ under the condition of the mix proportion keeping unchanged, and the peak strength increases more obviously with the increase of confining pressure.

2) Also, the organic curing agents can effectively improve the strain softening and strain hardening properties of heavy metal contaminated soil, make the dilatancy and contraction characteristics of heavy metal contaminated soil stable under different confining pressures, and enhance the stability of soil mass.

3) The hyperboloid volumetric strain constitutive model of heavy metal contaminated soil does not have the defect of the single yield model that can not describe strain critical state from dilatancy to contraction of the soil mass. The numerical fitting results are close to the experimental results, which indicate that this model has advantages in describing volumetric strain characteristics of heavy metal contaminated soil.

\section{Brief introduction of Author}

CHENG Feng (1980-), male, Ph.D, Professor, is mainly engaged in the research of environmental geotechnical engineering and foundation treatment. Email: chf46629@126.com.

\section{"Corresponding author}

WEI Cheng (1989-), male, Master, is mainly engaged in the research of geological engineering and geohazard prevention. Email: balckfire513@126.com.

\section{Foundation projects}

Natural Science Foundation of Guangxi Province of China, Project Funded by Ten-Hundred-Thousand Talents Program of Guangxi.

\section{References}

1. Pan, C., Liu, S.Y. (2019) Experimental research on thermal conductivity characteristics of heavy metal contaminated soil. Journal of Southeast University (Natural Science Edition), 49(2): 362-368.

2. Cao, Z.G., Zhang, D.W., Liu, S.Y. (2013) Experimental research on durability of solidified lead-contaminated soils under wetting-drying cycles. Rock and Soil Mechanics., 34(12): 3485-3490.

3. Huang, M., Chen, C.H., Yang, H.Z., Mao, Z.P., Li, Y.B. (2013) Effects of Two Typical Regulating Agents on Cadmium Fractions in Soils Contaminated by Cadmium. Journal of Wuhan University of Technology., 35(11): 132-137.

4. Wang, F., Shen, Z.T., Wang, H.L. (2018) Performances of cement-stabilised/solidified contaminated site soils. Journal of Geotechnical Engineering., 40(3): 540- 545.

5. Guo, Y.L., Cao, L.W., Huo, P. (2019) Strength and Micro-structure of Unsaturated Contaminated Soil under Two Stress Paths. Journal of Basic Science and Engineering., 27(3): 602-611.

6. Yin, X., Chen, J.J., Li, C. (2014) Impact of Compounded Chelants on Removal of Heavy Metals and Characteristics of Morphologic Change. Environmental Science., 35(2): 733-739.

7. Cui, J., Xue, W.p., Yan Z.Y., Wang, N. (2013) Repairing of heavy metals contaminated soil with 
different surfactants. Journal of Dalian Polytechnic University., 32(4): 279-282.

8. Li, K, Zhang, D.W., Cao, Z.G. (2019) Effects of carbonation on permeability characteristics of cement-stabilized/solidified lead-contaminated soil. Chinese Journal of Geotechnical Engineering., 41 (A2): 117-120.

9. Wu, G.L., Bo, Y.L., Du, Y.J., Wei, M.L., Xue, Q. (2019) Acid neutralization capacity, strength properties and micro-mechanism of $\mathrm{Pb}$-contaminated soils stabilized by alkali-activated GGBS. Journal of Geotechnical Engineering., 41(A1): 137-140.

10. Zha, F.S., Liu, J.J., Xu, L., Deng, Y.F., Yang, C.B., Chu, C.F. (2019) Electrical resistivity of heavy metal contaminated soils solidified/stabilized with cementfly ash. Rock and Soil Mechanics., (12): 4573-4580.

11. Zhu, C.P., Pan, L.Y., Yu, C., Wang, H., Jiang, J.F., Liao, R.P. (2018) Mechanical properties and microstructure characteristics of cement solidification pollution soil. Journal of civil, architectural \& environmental engineering., 40(3): 117-123.
12. Zhang, L.M., Wu, J., Liu, R.Q., Chen, W.Y., Zhao, J.L., Feng, Y.S., Xia, W.Y., Du, Y.J. (2017) Experimental study on the strength and environmental properties of $\mathrm{Ni}$ and $\mathrm{Zn}$ contaminated soil stabilized by SPB and SPC binders. Chinese Journal of Rock Mechanics and Engineering., 36(12): 3062-3074.

13. Zha, F.S., Hao, A.L., Xu, L., Liu, J.J., Cui, K.R. (2014) Experimental study of leaching characteristics of cement solidified and stabilized heavy metal contaminated soils. Industrial Construction., 44(1): 65-70.

14. Zhang, T.T., He, X.X., Wang, P., Xue, Q., Li, J.S. (2017) Influence of particle size and $\mathrm{pH}$ on stability of chromium contaminated soil and its mechanism analysis. Rock and Soil Mechanics., (A2): 82-88 .

15. Xu, Y.G., Cao, T., Luo, F. (2014) Wastewater Effluent Quality Prediction Model Based on Relevance Vector Machine. Journal of South China University of Technology (Natural Science Edition)., 42(5): 103-107. 